How to reference this article Mattarucco, G. (2018). Grammatica e pratica in alcuni manuali di italiano per stranieri del Seicento. Italica Wratislaviensia, 9(1), 123-137.

DOI: http://dx.doi.org/10.15804/IW.2018.09.07

Giada Mattarucco

Università per Stranieri di Siena

\title{
GRAMMATICA E PRATICA IN ALCUNI MANUALI DI ITALIANO PER STRANIERI DEL SEICENTO
}

\author{
GRAMMAR AND PRACTICE IN SOME 17 ${ }^{\mathrm{TH}}-\mathrm{CENTURY}$ \\ HANDBOOKS OF ITALIAN FOR FOREIGNERS
}

\begin{abstract}
The $17^{\text {th }}$-century handbooks of Italian for foreigners are rather different from one another in content, organisation, and size. Each author maintains that his book is better than the others', and although these statements have a promotional aim in general, these differences give us a glimpse of important questions that are the subject of discussion to this day, i.e., the varying emphasis on either norm or usage; models and examples to be proposed; and the weight to be given to the various components. While many authors deal with travels and travellers, Pierre Soulas, in his Grammaire et instruction pour comprendre en bref la langue italienne (1616), written for French students, states that grammar is more important than practice, and he considers himself a good teacher, even if he never set foot in Italy: he believes that sound rules and clear explanations allow for a greater advancement in two or three months than practice alone in one year. Girolamo Buoninsegni, on the other hand, boasts of a wealth of experience with his German students at Siena University, for whom he writes I primi principi della grammatica toscana (1618), which contains the basis of the language and starts from the very beginning. Lorenzo Franciosini, a native teacher but a Spanish scholar as well, is the author of several volumes and addresses even those "who in passing and, as it were, ready to run away, wish to learn the Tuscan language" (Fax linguae italicae, 1628).
\end{abstract}

Keywords: $17^{\text {th }}$-century handbooks, Italian for foreigners, Pierre Soulas, Girolamo Buoninsegni, Lorenzo Franciosini 


\section{PREMESSA}

T a grammaticografia e la storia degli insegnamenti linguistici, come del resto altri ambiti di ricerca in prospettiva diacronica, possono sia mettere in risalto le novità dei vari fenomeni e momenti, sia, all'opposto, avvalorare l'idea che, in definitiva, non vi sia "nulla di nuovo sotto il sole". Nella maggior parte dei casi appare preferibile e più equo pensare di ritrovarsi a metà strada tra i due poli. Ricorderò, dunque, alcuni manuali secenteschi di italiano lingua straniera, tentando di collocarli nel periodo e nel contesto cui appartengono e nel contempo di evidenziare in essi elementi e questioni tuttora centrali nella didattica delle lingue e dell'italiano in ispecie.

Ho già esaminato altrove (cfr. Mattarucco, 2003) manuali di quell'epoca, come l'Institution de la langue florentine et toscane di François Guédan (1602), rivolta a "persone d'honore, desiderose di saper la detta lingua, e haverla familiare, tanto per rispetto di ben leggerla, prononciar, scriver, e intenderla, quanto per comporre e tradurre i libri Italiani in Francese, e scambievolmente i Francesi in Italiano", come si dice nella dedicatoria a Maria de' Medici, alludendo alle varie abilità e competenze passive e attive della lingua sia scritta, sia orale, e al metodo grammaticale-traduttivo. Nell'epistola Al lettore benevolo Guédan (1602, pp. 10 sgg.) riassume le motivazioni per cui l'italiano, identificato con il toscano e in particolare con il fiorentino, viene studiato e merita d'esserlo - per diletto o addirittura in funzione antidepressiva, per utilità culturale ma anche "per il commercio e conversatione" tra Francia e Italia - e, lasciata da parte l'eccezione della "scienza infusa", come a Pentecoste, si concentra sull' apprendimento ordinario, "per travaglio, industria e ingegno". Guédan (1602) si basa sul canone dei buoni autori, classici di moda all'epoca, come Petrarca, Ariosto e Tasso, e perfino il predicatore piemontese Panigarola, il quale aveva a sua volta dovuto risciacquare i panni in Arno (Marazzini, 1993, pp. 102-105). Riferendosi quindi anche all'italiano vivo, che è utile sapere in maniera da non aver bisogno di interpreti per visitare la Penisola, Guédan (1602, pp. 11-12) menziona inoltre una lunga serie di mete di viaggio per i gentiluomini 
francesi, da Roma, a Napoli, a Venezia, a Firenze, a Siena, a molte altre "bellissime cittadi".

Ritroviamo il binomio lingua letteraria e fiorentino vivo anche nella Grammaire italienne mise et expliquée en François di César Oudin, "Secretaire Interprète du Roy, és langues Germanique, Italienne et Espagnolle": a parte poche modifiche e aggiunte, come un riferimento all'Orlando fuorioso, la grammatica di Oudin (1610) non è altro che una rielaborazione o traduzione degli Institutionum florentinae linguae libri duo di Eufrosino Lapini (1569), sacerdote, precettore e letterato fiorentino che aveva composto il proprio manuale, poi destinato ad ampia fortuna, quando si trovava all'estero per insegnare il toscano ad alcuni gentiluomini tedeschi.

\section{PER SOULAS, VAL PIÙ LA GRAMMATICA CHE LA PRATICA}

Pierre Soulas era un oscuro "Interprete és langues Françoise et Italienne", presumibilmente della zona di Poitiers, cui appare confinata la sua Grammaire et instruction pour comprendre en bref la langue italienne, edita nel 1616 appunto in tale città e forse mai diffusa altrove, visto che l'unico esemplare a noi pervenuto si trova ancora nella Bibliothèque municipale di Poitiers. ${ }^{1}$ L'opera è dedicata a "Henry Louis Chastaigner de la Roche-Pozay, tres-digne Evesque de Poictiers", un teologo ed erudito francese, italianisant e poliglotta, nato a Tivoli e trasferitosi a studiare a Leida, sotto la guida di Joseph Justus Scaliger. ${ }^{2}$ Nella dedicatoria, l'autore ammette di non conoscere l'illustre vescovo se non per fama e racconta d'aver solo di recente e su richiesta di alcuni gentiluomini affiancato l'insegnamento dell'italiano a quello della "langue Françoise aux estrangers". Presenta quindi il manuale come una sorta di dispensa, predisposta per nobili allievi in mancanza di altri strumenti adatti, reperibili in un ambiente di provincia ("en ces quartiers"), lontano dai luoghi

1 Cfr. Bingen, 1987, p. 223 e i dati nel Catalogue collectif de France (CCFr, 2018).

2 Su Henri-Louis Chasteigner de La Rochepozay (variamente scritto) si veda per esempio la scheda nell'archivio digitale dell'Istituto per il Lessico Intellettuale Europeo e Storie delle Idee (ILIESI, 2017). 
dove l'editoria italiana ferve come Parigi o Lione (se prendiamo alla lettera le parole che giustificano la pubblicazione).

Sempre nella dedicatoria e sempre in forma di excusatio non petita, Soulas fornisce altri particolari su di sé e sulla propria opera: sa d'essere esposto alle critiche, poiché non si è mai recato in Italia, ma sostiene che altri suoi colleghi, i quali hanno soggiornato nella Penisola due o tre anni se non di più, riescono magari a parlare la lingua italiana piuttosto bene, ma non sono capaci di spiegarne le strutture, come invece lui stesso è in grado di fare, su solide basi. Nell'insegnamento pone dunque al di sopra di tutto l'esperienza e la tecnica, di cui rivendica il pieno possesso, avendo a sua volta imparato l'italiano e il metodo da un ottimo maestro, peraltro egli sì toscano madrelingua. Soulas sostiene insomma che un buon apprendimento guidato, ovvero quello garantito da lui e dal suo testo, permetterebbe in due o tre mesi risultati migliori dell'apprendimento spontaneo in un anno intero.

In una simile prospettiva, fondamentale risulta la lettura dei buoni autori, come si desume già dal titolo o sottotitolo (Grammaire et instruction [...] recueillie de plusieurs bons Autheurs Italiens) e come viene ribadito nell'epistola introduttiva, attraverso la consueta immagine delle api che producono il miele succhiando il nettare dai fiori più profumati. Soulas dice d'aver messo insieme il proprio manuale a partire dalle fatiche di eccellenti autori ("de plusieurs bons et excellents autheurs") e a beneficio di quanti desiderano conoscere questa lingua eccellente ("pour les curieux d'une langue si excellente et polie comme est l'italienne"). Non stupisce allora che Petrarca sia menzionato in uno dei componimenti d'occasione in apertura, il sonetto in italiano di Bernerio della $\mathrm{Bruzza}^{3}$, figuri poi nella piccola antologia in appendice (cfr. più avanti) e sia comunque il solo autore citato all'interno della grammatica (p. 7v), per il verso "vive in speranza debile e fallace" (RVF, XXI, 6: esempio del fatto che in poesia non sempre si ricorra alla prostesi di $i$ davanti a $s$ complicata). Per il resto, nel corso del manuale, parecchie varianti sono segnalate come poetiche, ma senza citazioni o riferimenti

3 Gli altri due componimenti sono l'uno in latino, di "Stephanus Rifaldus", l'altro in francese, firmato "De La Coindrie". 
precisi (si vedano autoritate, rege, virtute, p. 4r; sovra, sovrano, ovra per sopra, soprano, opra, p. 5v; haggio, p. 13r; gire per andare o faccio in luogo del toscano fó, p. 22r; devo e deo anziché debbo e deggio, p. 23r; eccetera).

Soulas ricorre soprattutto a exempla ficta, di memoria classica (frasette quali Socrate fu piu savio di Diogene, pp. 10v-11r; da Platone laudasi / si lauda Socrate, p. 27r), d'ambito religioso (Signor Dio, io son contento d'un tal favore, p. 6r; leggo il vangelio di San Giovanni, p. 32r; e simili), o comunque di stampo edificante (io vorrei la pace non la guerra, p. 33r; hoggidi ho fatto il mio dovere, p. 35v), in particolare sul tema dello studio stesso (io ho sempre honorato / honorati $i$ dotti, p. 32v; imparare a leggere o scrivere, p. 34r). Dà spazio anche a un minimo di fraseologia spicciola (dammi del pane, versa qui dell'acqua, va per del vino, ecco del fromaggio [sic], con le alternative senza partitivo dammi il pane, ecco'l fromaggio e porta cuà [sic] il vino per il quale sei andato, p. 32r). Contempla perfino esempi oggi impensabili, come il mio padre e la mia madre sono morti (p. 28v) o Io vorrei m'essere nel Cielo (p. 34r).

L'impianto è tradizionale: si inizia con Des lettres, et de leur prononciation (pp. 1r sgg.), per passare alle parti del discorso (pp. 7v sgg.), ovvero, nell'ordine: articoli, nomi, che includono gli aggettivi, pronomi, verbi, con qualche approfondimento nella sezione La construction de parties d'Oraison, dove si parla anche delle parti invariabili: Adverbes, Prepositions, Interjections (pp. 35r sgg.). Ci troviamo di fronte a una grammatica normativa e sintetica, a volte un po' imprecisa: si veda l'incipit in cui Soulas elenca le venti lettere italiane, dalla $a$ alla $z$ (o ventuno con la $x$ ), e ne considera semplicisticamente i suoni uguali a quelli del francese o del latino, lasciando irrisolte molte questioni: per esempio, si limita a definire la e brefve, 'breve', in fine di parola, uguale al francese in riverentemente e lunga in Ré, Poté (p. 1v), mentre non parla affatto della $o$. Per i pronomi soggetto di terza persona, cioè al nominativo, secondo il modello latino, registra egli, ei, e' al maschile, ella al femminile, con eglino, ei, e', essi / ellene, esse al plurale, riservando lui, lei e loro ai casi obliqui (pp. $11 \mathrm{v}-12 \mathrm{r}$ ). Anche per il periodo ipotetico prevede solo esempi canonici come se io lo vedessi, io lo conoscerei, 
s'egli l'havesse veduto, l'havrebbe conosciuto, oppure s'io fossi prencipe io caccierei (pp. 32v-33r). Spesso Soulas se la cava con uno schema e poche righe di commento: si prenda il paragrafo Des Noms diminutifs (pp. 9v-10r), in cui il prospetto, con forme propriamente diminutive in -ino (fanciullino), -ello (velarello, pazzarello) e così via, accrescitive (scioccone) o peggiorative ("Les noms qui signifient chose mechante [...] comme giovine, giovinaccio [...] filosofo, filosofasto" [sic]), è accompagnato dall'osservazione seguente: "On faict quelquesfois d'un diminutif, un autre diminutif, comme pino [sic], pinuccio, cagnuolo, cagnuolino etc.".

Oltre alla grammatica (pp. 1r-37v), il manuale contiene una piccola antologia di letture (pp. 38r-48v). La prima di esse (pp. 38r-44r) è un volgarizzamento in prosa: Lo specchio del Ragionamento Vangelico, overo lo Stimolo d'Amore, tradotto di Latino in Italiano, dal R. P. F. NICOLO AURIFICO Senese Carmelitano, una meditazione di Sant'Anselmo che si legge nella Seconda parte delle Meditationi di diversi Dottori di Santa Chiesa, tradotte e pubblicate dal carmelitano Nicolò Buonfigli ${ }^{4}$ (Buonfigli 1583, pp. 49-67). Il secondo testo in appendice (Soulas 1616, pp. 44r-46v), come sopra accennato, è di Petrarca: Il Trionfo della Divinità, ovvero tutti i 145 versi del Triumphus Eternitatis. Come terza e ultima lettura (Soulas 1616, pp. 46v-48r) viene infine proposto sotto il titolo di Oratione ad Iddio un componimento in terzine:

Potentissimo Dio del sommo et imo, / Tu che creasti il Ciel, la terra e'l Mare, / Gli Angeli de la luce, e l'huom di limo / Tu che nel ventre vergine incarnare / Per noi volesti padre ognipotente, / Et nascere, e morire, e suscitare / [...] Ascolta padre l'humil prigo [sic] mio / Che supplice e divoto a te ne vegno / A te che ti festi huom per farme Dio / [...]

Benché non venga detto da Soulas, che riporta il testo senza menzionarne né l'autore né la fonte, si tratta di un capitolo scritto da Petru Cercel (1545-1590). Quest'ultimo era figlio di un voivoda valacco, era stato mandato da ragazzino a Costantinopoli, pegno vivente agli Otto-

4 Buonfigli sarebbe nato a Siena intorno al 1529 e morto nello stesso luogo nel 1603: cfr. la scheda OPAC SBN (2018). 
mani, e da lì si era poi recato in Europa, in particolare in Italia e in Francia, dove aveva intessuto relazioni politiche e culturali grazie alle quali aveva ottenuto il trono della Valacchia, conservato però solo per due anni (cfr. Vranceanu Pagliardini, 2017). Appunto Petru Cercel è al centro del secondo dei Dialoghi piacevoli di Stefano Guazzo (1586, pp. 14r sgg.), intitolato Del Prencipe della Valacchia Maggiore: un interlocutore, Francesco Pugiella, si proclama pronto a seguire il Principe straniero e, illustrandone i meriti al "Cavalier Guazzo" (l'altro interlocutore, fratello di Stefano), cita tutto il capitolo in terzine (Guazzo, 1586, 25v-26v). Petru Cercul aveva infatti imparato l'italiano, lingua in cui scriveva rime e lettere e che adoperava anche con i suoi protettori Henri III e Caterina de' Medici, e proprio durante il soggiorno in Francia aveva composto questo capitolo noto anche come Inno al Creatore. In chiusura del suo manuale, Soulas riporta quindi, forse senza nemmeno saperlo, un saggio di poesia italiana opera di uno straniero: un testo che ben rappresenta l'importanza dell'italiano nelle corti e tra gli umanisti dell'epoca, oltralpe nonché nel Mediterraneo e nella penisola balcanica.

\section{VOCI DEL MAESTRO E DEL POPOLO IN BUONINSEGNI (1618)}

Un'altra grammatica che mi sembra interessante ricordare è quella, di poco posteriore, di Girolamo Buoninsegni (1618). Senese, elogiato nelle Pompe di frate Isidoro Ugurgieri Azzolini (1649), membro dell'Accademia dei Filomati, Buoninsegni non aveva ottenuto di succedere al suo maestro Diomede Borghesi nella cattedra di toscana favella (che, com'è noto, era stata istituita a Siena nel 1588-89), limitandosi a supplire Celso Cittadini in tale ruolo e a fungere da lettore della nazione tedesca (cfr. Rossi, 1911, p. 392). Ci è pervenuta, tra il resto, una sua Orazione intorno alle lodi degli Insegnatori delle Scienze e dell' Arti e di chi bene appararle procura, pronunciata a San Domenico il 6 novembre 1612 "coll'occasione del ritorno agli studij" a Siena - oggi diremmo per l'apertura dell'anno accademico - e pubblicata lo stesso anno (Buoninsegni, 1612). Ricordiamo inoltre l'orazione da lui pronunciata il 4 novembre 1614, sempre a Siena, nella Chiesa della Madonna di 
Provenzano, e stampata l'anno dopo: in questo discorso Buoninsegni (1615, pp. 5 sgg.) da un lato pungola gli studenti, per risvegliarli "dal pigro sonno dell'ozio, sopravvenuto forse nella mente d'alcun di loro per le passate vacanze", dall'altro lato però ne prende le difese, convinto che l'inclinazione naturale vada sempre rispettata nei ragazzi. I maestri dovrebbero dunque aiutare gli allievi a perfezionarsi attraverso gli studi, mentre sono da biasimare $\mathrm{i}$ padri che pretendono di scegliere al posto dei figli:

Quanti se ne han veduti, stimolati dalla paterna ambizione entrar negli studi delle leggi $[. .$.$] o non v'han fatto alcun frutto, o se pur ve l'han fatto è stato$ leggiero [...] Doverebbono i vostri Padri per quel zelo che son tenuti d'haver del vostro bene, non secondo'l lor gusto, ma secondo la vostra inclinazione alle discipline impiegarvi.

Doverebbono (ardisco di dir) le leggi, proibir, ch'un giovane ad altr'arte fusse'ndirizzato, che a quella, di cui le radici ha poste in lui la Natura. (ivi, pp. 9-13)

Non stupisce quindi che il Buoninsegni fosse amato degli studenti. Perciò, i suoi Primi principi della grammatica toscana (1618) sono dedicati a "Teodorigo A. Quitzow", vale a dire Dieterich von Quitzow (cfr. Weigle, 1962, pp. 219 e 449), “Consiliario dell'Inclita / Nazione Alemanna, / ed a tutti / gli Illustrissimi Sig. Tedeschi / dimoranti in Siena". La dedicatoria rivela che Buoninsegni da circa dodici anni dava lezione a questi ultimi: egli adduce la consueta scusa d'essersi risolto a pubblicare la propria opera per le sollecitazioni degli allievi, "d'oltre a' monti [...] venuti in questa città" appositamente per imparare il toscano; si vanta quindi d'esser riuscito attraverso tali regole a insegnare a "bene e correttamente scrivere e favellare" perfino a coloro che non avevano "niuna contezza" della lingua, cioè ai Principianti (come li definisce poi in altri punti, con terminologia più moderna), quando allo zelo del maestro "s'è congiunta la diligenza loro".

Anche la grammatica di Buoninsegni (1618) segue l'ordine tradizionale: prima ortografia e pronuncia, poi morfosintassi, attraverso la canonica trattazione delle nove parti del parlamento: Articolo, Nome, Pronome, Verbo, Participio, Avverbio, Preposizione, Interiezzione, 
Congiunzione, sulla quale si chiude l'opera, priva di testi di corredo. Rispetto al manuale di Soulas, che non è madrelingua e insegna italiano all'estero, ci sono molte altre significative differenze: il lettore senese è forte della propria esperienza d'insegnamento a stranieri immersi nella lingua toscana e iscritti allo studio senese e fa capire che le regole da sole non bastano: gli allievi devono fare pratica, imparando non solo dalla viva voce dei maestri, ma anche dall'uso quotidiano, dal contatto con la gente. Buoninsegni sottolinea inoltre l'importanza della gradualità, il che implica attenzione a elementi e fenomeni che a qualcuno potrebbero sembrare trascurabili, ma che per discenti d'altra lingua non sono affatto scontati, “essendo impossibile, che l'edifizio del ben'apprendere stia'n piedi senza la base della fermezza, e pienezza delle regole, e della notizia di simili minute, e piccole cose" (1618, p. 9).

L'approccio pragmatico emerge fin dall'inizio del manuale, quando Buoninsegni (ivi, pp. 11-12) spiega che le vocali $i, u$ sono di semplice suono, come lui le chiama, "perché sempre nel pronunziarle si senton l'istesse", mentre $e$, o sono vocali di doppio suono "perché in due modi s'odon nella nostra favella, cioè o chiuse, come mele, meglio, torre, roc$c a$, od aperte, come nelle medesime voci, ma con altro significato" (significato che peraltro non spiega). Prosegue quindi:

Dell'uso de' qua' due suoni si dan molte regole, ma con molti capi, e con infinite eccezzioni, di modo che per minor briga, e men confusione, è più facil cosa l'imparargli da' maestri, e dal Popolo per uso, che dalle osservanze, e dalle regole, nondimeno posson generalmente osservarsi le cose infrascritte.

Si pronunzian sempre chiuse nella fin delle voci, quando son senza l'accento; ma quand'han l'accento sempre aperte.

In alcune particelle nondimeno, che essendo d'una sillaba, naturalmente han l'accento, come trattando degli accenti s'insegnerà, la regola fallisce imperciocchè si pronuncian chiuse, fe. Re. se. che. me. te. e simili.

La $e$. sempre si pronunzia stretta fra la $m$. e la $n$. mente. santamente. mento. e sì fatti. (p. 12)

Per i pronomi personali di terza persona, negli specchietti suddivisi come di consueto in base ai casi, alla latina, sono previsti al nominativo Egli, Eglino per il Genere del Maschio ed Ella, Elleno per il Genere 
della Femmina, per gli altri casi lui, lei, loro (p. 35). Quanto al periodo ipotetico, vengono forniti esempi come "S'io havessi denari, haverei ogni cosa" (p. 64) o "s'io havessi havuta l'arme, non haverei havuti tanti pericoli" (p. 66). Molto ricca l'esemplificazione degli alterati (p. 49): "Que' nomi, che con una voce sola mostran la piccolezza della cosa, e diminutivi son detti, han più fini nella nostra favella; impercocchè un positivo, che per esempio sarà casa. havrà tutti questi diminutivi casetta. casarella. casarellina casina. casinuccia. casarellinuccia. casellina. casellinuccia [...]". A questi si aggiungono "alcune voci" per indicare "la grandezza della cosa, maggiore della comune; e di queste ancora varie son le terminazioni. Vecchio. vecchiotto. vecchione. vecchiarellone. e sì fatti, i quali molto servono alla brevità della nostra favella" e i nomi che esprimono "disprezzo, e mostran la viltà della cosa. In accio, od accia [...] come puttanaccia. donnaccia. furfantaccio. ladraccio. e sì fatti, che per ingiuria si dicono tutto'l giorno, e significano scherno, e disprezzo". Buoninsegni contempla quindi, oltre alle "scritture de' nostr'huomini", l'uso vivo e le espressioni che "tutto'l dì s'odono nella voce del nostro popolo", incluse le voci più volgari (p. 49).

\section{USO E REGOLE IN FRANCIOSINI (1638)}

Consideriamo infine uno dei manuali d'italiano di Lorenzo Franciosini, che nelle sue stesse opere si presenta come nativo di Castelfiorentino e "Professore in Siena della Lingua Italiana, e Spagnuola": noto forse soprattutto per la prima traduzione italiana completa del Don Chisciotte pubblicata tra 1622 e 1625, cura un Vocabolario italiano e spagnolo (1620), una Grammatica spagnuola e toscana (Franciosini, 1624) ${ }^{5}$ e la versione toscana di alcuni dialoghi $(1626)^{6}$, rifacendosi largamente al lavoro di un altro grammatico e lessicografo del XVII secolo, César Oudin (cfr. Del Bravo, 1998). In particolare, all'insegnamento dell'italiano

5 Si veda l'edizione critica in rete, a cura di Félix San Vicente (2016).

6 Si tratta di dialoghi destinati originariamente da Minsheu all'insegnamento dello spagnolo in Inghilterra e poi ripresi e adattati da molti altri autori, prima e dopo Franciosini (cfr. Mattarucco, 2015). 
a stranieri egli dedica due testi: De particulis Italicae orationis (Franciosini, 1637) e quello che qui ci interessa, Fax linguae Italicae (1638), poi riedito più volte, anche in forma di compendio (1644).

La grammatica del 1638, che ha ricevuto l'imprimatur fin dal 1636, è dedicata al Principe Leopoldo di Toscana, colto e giovane governatore di Siena, e, come altri manuali destinati a un pubblico internazionale, scritta in latino, a parte l'epistola introduttiva che appare in duplice versione: prima in italiano (A chi leggerà) e poi in latino anch'essa (Lecturo). In tale epistola, Franciosini (1638, pp. 3 sgg.), con la canonica esibizione di modestia, definisce la propria un'operetta (o opusculum) conforme alla "intenzione di brevemente, e presto insegnar'a coloro, che alla sfuggita, e stivalati (per così dire) voglion' imparar la favella Toscana", anche se in realtà il volume è assai corposo (si compone di ben 646 pagine). L'autore esprime inoltre fin da subito la convinzione che i destinatari non condanneranno la scelta di accogliere forme d'uso comune, magari in contrasto con la tradizione codificata ma "ammesse" da "infiniti Scrittori Toscani" e da lui stesso adoperate

essendo i vocaboli, che comunemente si parlano, come la moneta, che giornalmente si spende, la qual, non per esser di buona lega è ricevuta, ma per esser corrente nella piazza dell'uso, nella giurisdizion del quale perde la regola ogni ragione. (ivi, p. 4)

Franciosini giustifica, quindi, una serie di varianti: Altrimenti anziché Altramente, Continuo per Continovo, Nuovamente in luogo di Novamente e simili. Da toscano madrelingua, ritiene che l'uso sia superiore alla norma astratta e presenta la propria grammatica come descrittiva più che prescrittiva.

Ampio spazio hanno la fonologia e la morfosintassi, sempre secondo l'ordine tradizionale, dalle parti variabili a quelle invariabili: le forme vengono via via illustrate e tradotte in latino, di solito prima in prospetti e tavole, poi attraverso una serie di osservazioni. Così nel Caput I, Franciosini $(1638$, p. 1) elenca le lettere dell'alfabeto e dice: "Quarum sonus, licèt viva docentis voce melius, quam praeceptis exprimi possit"; cionondimeno sintetizza alcune peculiarità della pronuncia. Per la $e$ distingue dunque un suono ora aperto, come in bello, 
mensa, remo, ora chiuso, come in refe, aréna, generazione, stella ( $\mathrm{p}$. 5), mentre scrive che $o$ "Pronunciatur aliquando remisse, ut botta, aliquando vero stricte, ut botte" (p. 10). Franciosini (ivi, pp. 75 sgg.) riporta negli specchietti dei pronomi di terza persona egli, ei, e' / eglino ed ella / elle, elleno al femminile per il nominativo, inserendo lui, lei, loro per gli altri casi, ma poi, alla stregua di molti grammatici cinquesecenteschi, contempla varie possibilità: essi, esse al plurale, lui e lei con il gerundio o "Ella \& vulgo lei" come allocutivo. Nel paragrafo De Nominibus diminutivis Franciosini (ivi, pp. 42-43) ricorda i suffissi più comuni "apud Etruscos", ovvero "ello, ut arditéllo", "etto, ut giovanétto", "ino, ut libbriccíno", "uccio, ut homúccio", "uzzo, ut pensierúzzo" (segnando anche gli accenti tonici). Osserva quindi come da un diminutivo possa formarsene un altro, nella serie casétta, casettína, casupolína, casellína; rileva la valenza "blanditiva" di forme quali fanciullíno, bellíno, ragazzíno e viceversa il significato spregiativo di -accio in casáccia, homáccio, donnáccia, libráccio e di -ame in gentáme, contadináme eccetera. Per il periodo ipotetico dell'irrealtà, contempla anche l'uso dell'imperfetto indicativo: "Hoc eodem imperfecto indicativi utuntur frequenter Etrusci pro plusquam perfectum subiunctivi praemissa particula se, ut se tu facévi quello, ch'io ti dissi, non ti succedéva questo pro se tu havessi fatto etc. non ti sarebbe succedùto etc." (p. 158). Talvolta, la grammatica sconfina nel dizionario: per esempio, per il verbo havere (pp. $162 \mathrm{sgg}$.) è riportato un lungo elenco di significati, con polirematiche e modi di dire (sempre con i rispettivi equivalenti in latino): haver pensieri, haver più cervello degli altri, haver da fare, haver ragione e così via.

\section{CONCLUSIONI}

Per quanto possibile in un breve contributo come questo, le grammatiche sono state scelte e descritte in modo da dare un'idea della varietà di metodi e modelli proposti già nella prima metà del Seicento. Dal sia pur piccolo campione emergono infatti differenze in merito a opere, autori, destinatari, contenuti, dimensioni, ma anche elementi di continuità legati al canone letterario, al prestigio della varietà toscana e alla tradizione 
grammaticale stessa. Pierre Soulas, come poco prima François Guédan e César Oudin, è un francese che insegna in Francia e si rivolge ad allievi francesi, perciò le spiegazioni sono in francese. Invece Girolamo Buoninsegni è di Siena e dà lezione nella sua stessa patria a studenti stranieri, in particolare tedeschi, che frequentano l'ateneo cittadino: egli adopera quindi direttamente l'italiano come metalingua della grammatica. Anche Lorenzo Franciosini è un toscano madrelingua di area fiorentina e lavora a Siena, ma avendo in mente un pubblico più composito e internazionale, costituito da stranieri di passaggio in Italia, scrive il suo manuale in latino. Soulas pubblica un'opera più smilza, con non poche incertezze e imprecisioni: del resto, egli ammette di non aver mai messo piede in Italia e offre il fianco alla critica già mossa da Bembo a Fortunio, di "insegnare altrui quello che egli non sapea". Bisogna però riconoscere al maître di Poitiers alcuni meriti: primo tra tutti forse quello d'aver saputo scegliere validi modelli, sia rifacendosi all'esempio del bravo insegnante toscano (dal quale egli stesso ha imparato la lingua, come dice) sia allegando alla vera e propria grammatica testi di lettura non banali, che presumibilmente ben corrispondono alle esigenze dei destinatari. Buoninsegni e Franciosini hanno invece conoscenza sia della lingua, per loro materna, sia del metodo, a lungo sperimentato nell'insegnamento. Proprio per questo, sanno che il manuale non basta, ma sono necessarie agli apprendenti le vive voci dei maestri e le ancor più vivaci esperienze d'uso del toscano nella vita quotidiana, a contatto con la gente comune.

\section{BIBLIOGRAFIA}

Bingen, N. (1987). Le Maître italien (1510-1660). Bibliographie des ouvrages d'enseignement de la langue italienne destinés au public de langue française, suivie d'un Répertoire des ouvrages bilingues imprimés dans les pays de langue française. Bruxelles: Émile Van Balberghe.

Buonfigli, N. (ed.) (1583). Seconda parte delle Meditationi di diversi Dottori di S. Chiesa, novamente tradotte, e corrette dal R. P. F. Nicolò Aùrifico Buonfigli Senese Carmelitano. Venezia: Appresso i Gioliti. 
Buoninsegni, G. (1612). Orazione intorno alle lodi degl'insegnatori delle Scienze e dell'Arti e di chi bene appararle procura. Siena: Nella Stamperia di Matteo Florimi.

Buoninsegni, G. (1615). Orazione della forza della naturale inclinazione, E che nell'indirizzare i Giovani alle Scienze, ed all'Arti, a quella si vuol haver principalissimo riguardo. Siena: per gli Heredi di Matteo Florimi.

Buoninsegni, G. (1618). I Primi principi della grammatica toscana. Siena: per l'erede di Matt. Florimi.

CCFr. (2018). Catalogue collectif de France. Retrieved from goo.gl/8DA9bb.

Del Bravo, S. (1998). Franciosini, Lorenzo in Dizionario biografico degli italiani (vol. 50, pp. 155-157). Roma: Istituto della Enciclopedia Italiana.

Franciosini, L. (1624). Gramatica spagnola, e italiana. Venezia: Presso Giacomo Sarzina.

Franciosini, L. (1626). Dialogos apazibles, compuestos en Castellano y traduzídos en Toscàno / Dialoghi piacevoli, composti in Castigliano e tradòtti in Toscàno. Venezia: presso Giacomo Sarzina.

Franciosini, L. (1637). De particulis Italicae orationis. Florentiae: Typis novis Amatoris Massae, \& Socior.

Franciosini, L. (1638). Fax linguae Italicae. Florentiae: Typis novis Amatoris Massae, \& Socior.

Franciosini, L. (1644). Compendium facis linguae Italicae. Senis: apud Bonettos typis publicis.

Guédan, F. (1602). Institution de la langue florentine et toscane. Paris: Iean Gesselin.

Guazzo, S. (1586). Dialoghi piacevoli. Venezia: presso Gio. Antonio Bertano. ILIESI (2017). Lessici filosofici. Retrieved from goo.gl/zXD6Yv.

Lapini, E. (1569). Institutionum Florentinae linguae libri duo. Florentiae: apud Iunctas.

Marazzini, C. (1993). Storia della lingua italiana. Il secondo Cinquecento e il Seicento. Bologna: Il Mulino.

Mattarucco, G. (2003). Prime grammatiche d'italiano per francesi (secoli $X V I-X V I I)$. Firenze: Accademia della Crusca.

Mattarucco, G. (2015). Poligloto et alii: dialoghi piacevoli. In C. Bruno, S. Casini, F. Gallina \& R. Siebetcheu (eds.) Plurilinguismo / Sintassi (pp. 433-448). Roma: Bulzoni.

OPAC SBN. (2018). Catalogo del Servizio Bibliotecario Nazionale. Retrieved from goo.gl/saFaec. 
Oudin, C. (1610). Grammaire italienne mise et expliquée en François. Paris: Iean Gesselin.

Rossi, P. (1911). La prima cattedra di "lingua Toscana" (dai Ruoli dello studio senese 1588-1743). Studi senesi, XXVII (5), 345-394.

San Vicente, F. (2016). La tradición gramatical del español en Italia. La «Gramatica spagnola e italiana» de Lorenzo Franciosini. Estudio y edición crítica. Retrieved from epigrama.eu/public/index.

Soulas, P. (1616). Grammaire et instruction pour comprendre en bref la langue italienne. Poitiers: Iulian Thoreau.

Stammerjohann, H. (2013). La lingua degli angeli. Italianismo, italianismi e giudizi sulla lingua italiana. Firenze: Accademia della Crusca.

Ugurgieri Azzolini, I. (1649). Le pompe sanesi, o'vero, Relazione delli huomini, e donne illustri di Siena e suo Stato. Pistoia: nella stamperia di Pier' Antonio Fortunati.

Vranceanu Pagliardini, A. (2017). I motivi di una scelta: Stefano Guazzo e il "Prencipe della Valacchia Maggiore" come modello morale per la corte. Philologica Jassyensia, XIII, 1 (25), 261-273.

Weigle, F. (1962). Die Matrikel der Deutschen Nation in Siena (1573-1738). Tübingen: Max Niemeyer.

Riassunto: I manuali secenteschi di italiano destinati a stranieri sono alquanto vari per contenuti, impianto, dimensioni. Ciascun autore sostiene d'aver elaborato un testo migliore degli altri; si tratta certo in larga parte di proclami propagandistici, che lasciano però intravedere questioni importanti, tuttora dibattute: oscillazione tra norma e uso, modelli ed esempi da proporre, peso da attribuire alle varie componenti. Se molti manualisti parlano di viaggi e viaggiatori, viceversa Pierre Soulas, nella Grammaire et instruction pour comprendre en bref la langue italienne (1616), redatta per allievi francesi, afferma che la grammatica val più della pratica e si reputa un valido maestro pur non avendo mai messo piede in Italia: a suo avviso, buone regole e spiegazioni fanno progredire in due o tre mesi più di quanto non permetta in un anno l'uso da solo. Girolamo Buoninsegni invece vanta una lunga esperienza con gli studenti tedeschi dell'Università di Siena, a beneficio dei quali compone I primi principi della grammatica toscana (1618), con i rudimenti della lingua, a partire da zero. Lorenzo Franciosini, altro insegnante toscano madrelingua, ma anche ispanista, autore di diversi volumi, si rivolge perfino "a coloro, che alla sfuggita, e stivalati (per così dire) vóglion' imparar la favélla toscana" (Fax linguae Italicae, 1638).

Parole chiave: manuali secenteschi; italiano come lingua straniera; Pierre Soulas; Girolamo Buoninsegni; Lorenzo Franciosini 